\title{
Exact Output Response Computation of RC Interconnects Under General Polynomial Input Waveforms
}

\author{
L. M. PATNAIK ${ }^{\mathrm{a}, *}$ and SATRAJIT GUPTA ${ }^{\mathrm{b}}$ \\ ${ }^{a}$ Microprocessor Applications Laboratory, Indian Institute of Science, Bangalore, India, 560012; \\ ${ }^{\mathrm{b}}$ Dept. of Electrical Communication Engineering, Indian Institute of Science, Bangalore, India, 560012
}

(Received 20 February 1999; In final form 14 October 1999)

\begin{abstract}
Accurate output response computation of RC interconnects under various input excitations is a key issue in deep submicron delay analysis. In this paper, we present an exact analysis of output response computation of a distributed RC interconnect under input signals that are polynomial in time $\left(t^{n}\right)$. A simple, recursive equation that helps us to calculate the interconnect response under higher order polynomial inputs in terms of the lower order polynomial responses is derived. To the best of our knowledge, this is the first exact output response analysis of RC interconnects under generalized polynomial inputs.
\end{abstract}

Keywords: Interconnect modelling, modelling and simulation of digital systems, deep submicron systems, exact modelling of interconnects, RC interconnects

\section{INTRODUCTION}

Integration density and performance of integrated circuits (ICs) have gone through a stunning improvement in the last couple of decades. This has strongly influenced the complexity of systems that can be put on a chip. In fact, deep submicron $(<0.5 \mu \mathrm{m})$ fabrication lines give designers ample silicon "space" to put entire systems on chip. In view of this, the primary concern in VLSI design nowadays is to efficiently harness the available silicon area. One of the biggest bottlenecks towards achieving this is the increasing significance of interconnects in determining the performance of a system [2].

Interconnects in an integrated circuit or system introduce capacitive, resistive and inductive parasitics that can have a dominant influence on circuit operation in the form of coupling noise, ground bounce and substrate coupling. More importantly, these parasitic effects display a scaling behaviour that is different from the active blocks making up the rest of the circuit. Their effects increase as device dimensions are reduced and they dominate the performance in deep submicron technologies. This situation is aggravated by the fact that

\footnotetext{
*Corresponding author. e-mail: 1alit@micro.iisc.ernet.in
} 
improved manufacturing technologies lead to ever larger die sizes which, in turn lead to longer global wires. It is therefore of utmost importance to analyze and understand the dynamics of delay through interconnects.

Because of the reasons mentioned above, there has been considerable research effort over the years to accurately analyze simple interconnect structures both in terms of time-domain and transform-domain responses. Cascaded RC chain [1] is the most popular model for interconnect structures. Consequently, there has been a lot of research towards characterizing the RC interconnect structure.

Traditionally "Elmore delay" [3] is used as a metric for estimating delay through $\mathrm{RC}$ interconnects. Elmore delay is the first order time constant (also called the first moment of the impulse response) of the RC chain. However, the RC chain, in reality, is characterized by a number of time constants. The Elmore expression determines only the dominant one and thus represents a first-order approximation. At best, it is an upper bound on the RC interconnect delay. And although researchers have proved Elmore delay to be a bound on the $50 \%$ threshold delay for RC interconnects under input waveforms more general than step [4], it has been reported that the Elmore delay can be very different from Spicecomputed $50 \%$ delay. In fact, depending on the input slew time and driver resistance, the Elmore delay can be either greater or less than the Spice computed delay [5]. This has prompted researchers to look for more accurate and computationally efficient delay estimates through $\mathrm{RC}$ interconnects.

However till now most of the output response computation has been done for step and ramp inputs. This is equivalent to neglecting the effect of real-life wave shapes on delay computation. As pointed out in [6] this can lead to wrong delay measures in operation regimes where interconnect $\mathrm{RC}$ effects dominate.

In this paper, we present analytical expressions for output response of RC interconnects under input waveforms that are polynomial in time. It is shown that a recursive formula that helps us to calculate the output response for higher order input polynomial waveforms from the output response expressions for lower order input polynomial waveforms can be derived. Once we get the output response expression, the delay at a particular threshold voltage $(50 \%$ or $90 \%)$ can be obtained easily by numerical or graphical methods.

This paper is organized as follows. In Section 2 we present a survey of the work on RC interconnects. The output response computation formulae are presented in Section 3. Finally Section 4 concludes with a discussion on the future scope of the work. The two Appendices A and B contain the detailed derivation of results central to our output response computation.

\section{RC INTERCONNECT ANALYSIS - A SURVEY}

Broadly, the study of RC interconnects can be divided into two categories

- Accurate time domain or $s$-domain analysis of the interconnect structure which yields an analytic expression of the output voltage waveform. This is the method we will be concentrating on in our analysis.

- Moment-matching methods for reduced order $\mathrm{RC}$ interconnect transfer function synthesis where the RC interconnect transfer function is modelled as a reduced order rational function in $s$-domain. The rational function coefficients are obtained by moment-matching.

Accurate time-domain or $s$-domain analysis of $\mathrm{RC}$ interconnects can be done by treating the interconnect as a 2-port and obtaining the $s$ domain equations for voltage and current at the terminal side of the 2-port in terms of the voltage and current at the source side. Once we get the response in the $s$-domain using 2-port parameters, we can apply inverse transforms to get the response in the time-domain. However getting 
the inverse transforms might be difficult for arbitrary $s$-domain responses. So various simplifying approximations which give tractable expressions for the time domain response are made. This is the approach that is followed in most of the early derivations. A complete summary of these derivations is given in [7].

A complete time domain solution was given in [8]. However, they made the simplifying assumption of 'separability' which means that the voltage response function can be decomposed into separate functions of time and position. Recently researchers have reported exact expressions for output response of a semi-infinite RC line under step input [7]. In a subsequent paper, they extended their work to include ramp inputs [9]. A general approach has been presented to compute the time domain ramp response of finite $\mathrm{RC}$ lines. Closed form expressions of ramp input response have been presented for both capacitively loaded and open-ended finite RC lines. Another interesting work in this area is that of Rao [10].

\subsection{Limitations of the Previous Approaches}

However, all these approaches deal with a very restricted class of inputs-step and ramp. Real-life waveforms in VLSI circuits can be very different from these simple abstractions. This difference between real-life waveforms and the waveforms used for output response computation can be crucial for deep submicron delay estimation. Specifically, it has been shown that because of the increasing interconnect resistance, in state-ofthe-art designs the driver sees only a part of the total output net capacitance. This phenomenon is referred to as 'Resistance Shielding' [6]. Because of this, the waveform at the input of the interconnect shows a non-linear behaviour and this nonlinear waveform can strongly influence the delay of the RC interconnect. This increasing dependence of delay on waveform shape has been the motivation for us to look for analytical expressions of RC interconnect outputs under more general input waveforms than step or ramp. With this in mind, in this paper, we present analytical expressions of distributed semi-infinite RC interconnect response under input waveforms that are polynomial in time $\left(t^{n}\right)$. The reasons behind choosing a polynomial waveform are as follows:

- Any analytic function in its radius of convergence can be represented as a power series which consists of a summation of an infinite number of polynomials.

- Besides analytic considerations, the easiest way to model an arbitrary waveform is to approximate it as a polynomial expression whose coefficients can be determined by curve-fitting methods.

So once we have analytic expressions for $\left(t^{n}\right)$ type of polynomial inputs, we can approximate the response to an arbitrary waveform by summing up the appropriate polynomial $\left(t^{n}\right)$ responses. This serves as a more powerful framework for considering input waveforms more general than step or ramp. In fact there have been nonlinear waveform characterizations already in the literature [11]. In the next section, we present the details of our analysis.

\section{EXACT ANALYSIS UNDER POLYNOMIAL INPUTS}

In this section, the time-domain response of $\mathrm{RC}$ interconnects under polynomial input waveforms is analysed. We assume the following form for an $n$-th order infinite input polynomial waveform (Fig. 1),

$$
V_{\text {in }}(t)=\frac{V_{0}}{T_{R}^{n}} t^{n} u(t)
$$

where $T_{R}$ is the time at which the input waveform reaches the value $V_{0}$ and $u(t)$ is the unit step waveform that starts at time $t=0 . u(t)$ is the unit step waveform that starts at time $t=0$.

We consider the case of the semi-infinite RC line (Fig. 2) first. 


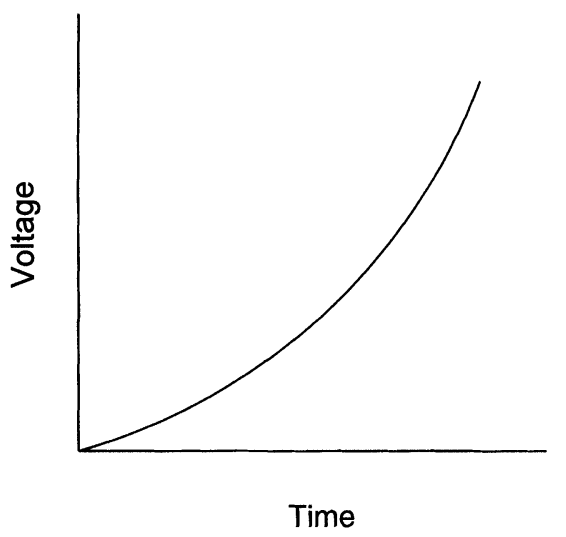

FIGURE 1 The infinite polynomial input waveform.

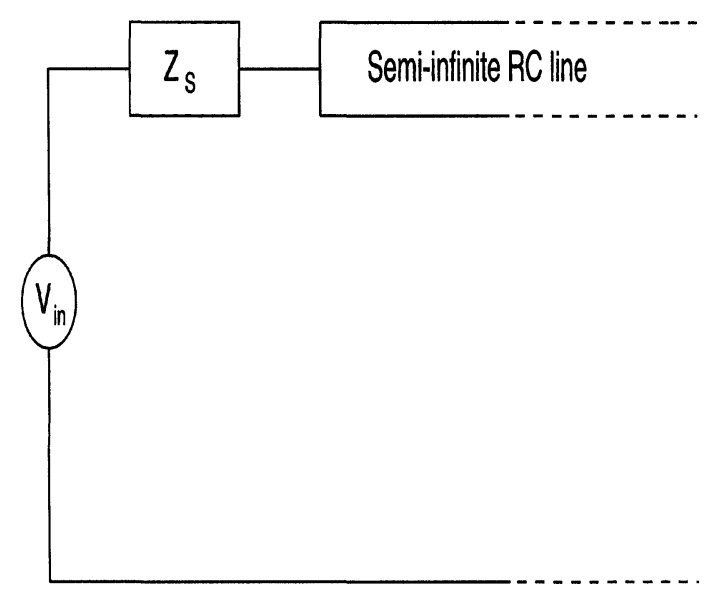

FIGURE 2 The semi-infinite RC line.

The diffusion equation for a distributed RC line is given by

$$
\frac{\partial^{2} v_{n}(x, t)}{\partial x^{2}}=r c \frac{\partial v_{n}(x, t)}{\partial t}
$$

where $r$ and $c$ are the per unit length resistance and capacitance respectively of the interconnect and $v_{n}(x ; t)$ is the output voltage waveform at a distance $x$ from the source at time $t$ under $n$-th order infinite polynomial input (i.e., a polynomial input of the form $\left(V_{0} / T_{R}^{n}\right) t^{n} u(t)$ as defined earlier).
The boundary conditions for the problem are:

$$
\begin{aligned}
& v_{n}(x, 0)=0 \\
& v_{n}(0, t)=V_{\text {in }}(t)=\frac{V_{0}}{T_{R}^{n}} t^{n} u(t)
\end{aligned}
$$

We denote

$$
w_{n, k}(x, t)=\frac{\partial^{k} v_{n}(x, t)}{\partial x^{k}} \text { for } k=0, \ldots, n
$$

Clearly $\quad w_{n, 0}(x, t)=v_{n}(x, t) \quad$ and $\quad w_{n, n}(x, t)=$ $\left(\partial^{k} v_{n}(x, t) / \partial x^{k}\right)$

Now the problem can be transformed into:

$$
\frac{\partial^{2} w_{n, n}(x, t)}{\partial x^{2}}=r c \frac{\partial w_{n, n}(x, t)}{\partial t}
$$

With the boundary conditions:

$$
\begin{gathered}
w_{n, n}(x, 0)=0 \text { for all } x \geq 0 \\
w_{n, n}(0, t)=n ! \frac{V_{0}}{T_{R}^{n}} u(t) \quad \text { for all } t \geq 0
\end{gathered}
$$

The solution to the transformed problem is given by [9]

$$
w_{n, n}(x, t)=\frac{n ! V_{0}}{T_{R}^{n}} \operatorname{erfc}\left(\frac{x \sqrt{r c}}{\sqrt{4 t}}\right)
$$

Let us denote $V_{0} \operatorname{erfc}(x \sqrt{r c} / \sqrt{4 t})=I_{0}(x, t)$ and let $I_{i+1}(x, t)=\int_{0}^{t} I_{i}(x, \tau) d \tau$ for $i=0,1,2, \ldots$

Then $v_{n}(x, t)$ is given by:

$$
v_{n}(x, t)=\frac{n !}{T_{R}^{n}} I_{n}(x, t)
$$

It can be shown that (Appendix-A)

$$
I_{2}(x, t)=\left(k_{1,2} t+k_{2,2}\right) I_{1}(x, t)-k_{3,1} t^{2} I_{0}(x, t)
$$

where $k_{1,2}=5 / 6, k_{2,2}=\left(x^{2} r c / 12\right), k_{3,1}=1 / 3$

The above result is proved in Appendix-A.

Further, it can be easily shown that, if

$$
\begin{aligned}
I_{m}(x, t)= & \left(k_{1, m} t+k_{2, m}\right) I_{m-1}(x, t) \\
& -k_{3, m} t^{2} I_{m-2}(x, t)
\end{aligned}
$$


then

$$
\begin{aligned}
I_{m+1}(x, t)= & \left(k_{1, m+1} t+k_{2, m+1}\right) I_{m}(x, t) \\
& -k_{3, m+1} t^{2} I_{m-1}(x, t)
\end{aligned}
$$

where

$$
\begin{aligned}
& k_{1, m+1}=\left(\frac{k_{1, m}+2 k_{3, m}}{1+k_{1, m}+2 k_{3, m}}\right) \\
& k_{2, m+1}=\left(\frac{k_{2, m}}{1+k_{1, m}+2 k_{3, m}}\right) \\
& k_{3, m+1}=\left(\frac{k_{3, m}}{1+k_{1, m}+2 k_{3, m}}\right)
\end{aligned}
$$

The above result is proved in Appendix-B.

Using Eqs. (7) and (8) we can recursively compute $I_{k}(x, t)$ for $k=3,4, \ldots$

Now, the response of a semi-infinite RC line under a $(n+1)$ st polynomial $\left(\left(V_{0} / T_{R}^{n+1}\right) t^{n+1} u(t)\right)$ is given by,

$$
v_{n+1}(x, t)=\frac{(n+1) !}{T_{R}^{n+1}} I_{n+1}(x, t)
$$

We substitute $m=n$ in Eq. (8) to get,

$$
\begin{aligned}
I_{n+1}(x, t)= & \left(k_{1, n+1} t+k_{2, n+1}\right) I_{n}(x, t) \\
& -k_{3, n+1} t^{2} I_{n-1}(x, t)
\end{aligned}
$$

For an $m$-th order input waveform the response is given by,

$$
v_{m}(x, t)=\frac{m !}{T_{R}^{m}} I_{m}(x, t)
$$

From Eqs. (9) -(11) we get,

$$
\begin{array}{r}
v_{n+1}(x, t)=\frac{(n+1)}{T_{R}}\left[\left(k_{1, n+1} t+k_{2, n+1}\right) v_{n}(x, t)\right. \\
\left.-\frac{n k_{3, n+1}}{T_{R}} t^{2} v_{n-1}(x, t)\right]
\end{array}
$$

where $k_{1, n+1}, k_{2, n+1}$ and $k_{3, n+1}$ are as defined earlier.

We can see from Eq. (12) that the response for the $(n+1)$ st order polynomial input can be derived in terms of responses for the $n$-th order and $(n-1)$ st order polynomials.
Since we have closed form solutions for responses to step, ramp [9] and quadratic input waveforms (Appendix-A), we can recursively generate exact expressions of higher order polynomial response waveforms.

Till now we have considered infinite polynomial input waveforms. Now we can derive the response for the more practical case of finite polynomial input waveforms (Fig. 3) (i.e., polynomial input waveforms that saturate to a fixed value after a finite amount of time).

We assume the finite polynomial input to saturate at a value $V_{0}$ after time $T_{R}$.

The input waveform expression in this case is given by:

$$
\begin{gathered}
V_{\text {in }}(t)=\frac{V_{0}}{T_{R}^{n}}\left[t^{n} u(t)-\left(t^{n}-T_{R}^{n}\right) u\left(t-T_{R}\right)\right] \\
=\frac{V_{0}}{T_{R}^{n}}\left[t^{n} u(t)-\sum_{k=1}^{n}{ }^{n} C_{k} T_{R}^{n-k}\left(t-T_{R}\right)^{k}\right]
\end{gathered}
$$

Let us denote the output response in the case of a finite polynomial input by $u_{n}(x, t)$.

From Eqs. (11), (12) and (14) we see that $u_{n}(x, t)$ is given by,

$$
u_{n}(x, t)=v_{n}(x, t)-\sum_{k=1}^{n}{ }^{n} C_{k} v_{k}\left(x, t-T_{R}\right)
$$

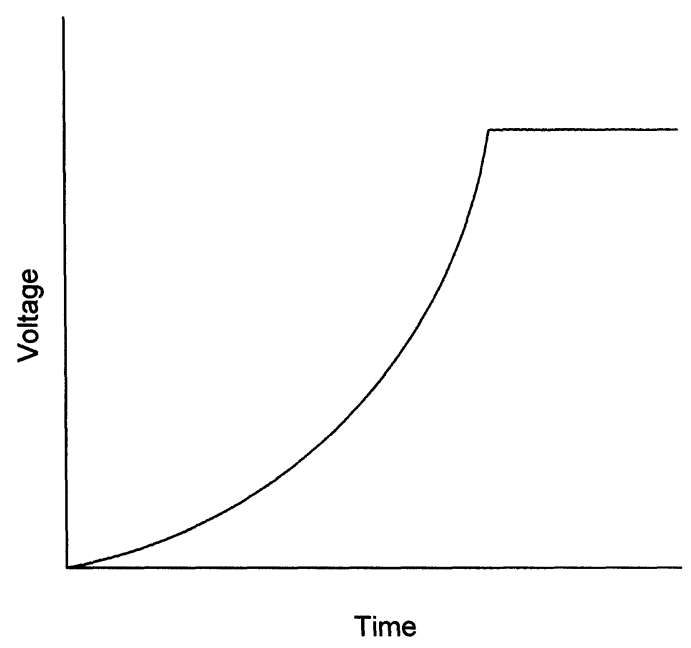

FIGURE 3 The finite polynomial input waveform. 
where $v_{k}(x, t)$ is the output response to the infinite polynomial input $\left(V_{0} / T_{R}^{k}\right) t^{k} u(t)$. It can be easily calculated using the iterative Eq. (12) given above.

With Eq. (15), we can derive the exact timedomain response of an $\mathrm{RC}$ interconnect under finite polynomial input waveform.

As mentioned before, we can represent any arbitrary waveform with an analytic representation as a power series. For practical purposes, we can truncate the series after a suitable number of polynomial terms. We can find the response separately for each polynomial term using the Eqs. (12) and (15) given above and sum the responses to get the final response waveform.

\subsection{Extension to the Case of Finite RC Lines}

Till now we have analyzed the response of a semiinfinite RC line under polynomial input waveforms. When we consider the practical case of a finite RC line with arbitrary load impedance (Fig. 4) the situation becomes different because of the load and source reflections. As a result, the voltage waveform at $(x, t)$ is a sum of the incident waveform and an infinite number of reflected waveforms.

Let $\Gamma_{l}(s)$ and $\Gamma_{s}(s)$ be the $s$-domain reflection coefficients at the source and the load ends respectively. Then

$$
\Gamma_{l}(s)=\frac{Z_{l}-Z_{o}}{Z_{l}+Z_{o}}
$$

and

$$
\Gamma_{s}(s)=\frac{Z_{s}-Z_{o}}{Z_{s}+Z_{o}}
$$

where $Z_{l}(s), Z_{s}(s)$ and $Z_{o}(s)$ denote the load, source and characteristic impedance of the line respectively.

In this case the expression for the output waveform in $s$-domain is given by [9]

$$
\begin{aligned}
U_{n}(x, s)= & V_{n}(x, s) \\
& +\sum_{k=1}^{\infty}\left[\Gamma_{l}^{k}(s) \Gamma_{s}^{k-1}(s) V_{n}(2 k L-x, s)\right. \\
& \left.\quad+\Gamma_{l}^{k}(s) \Gamma_{s}^{k}(s) V_{n}(2 k L+x, s)\right]
\end{aligned}
$$

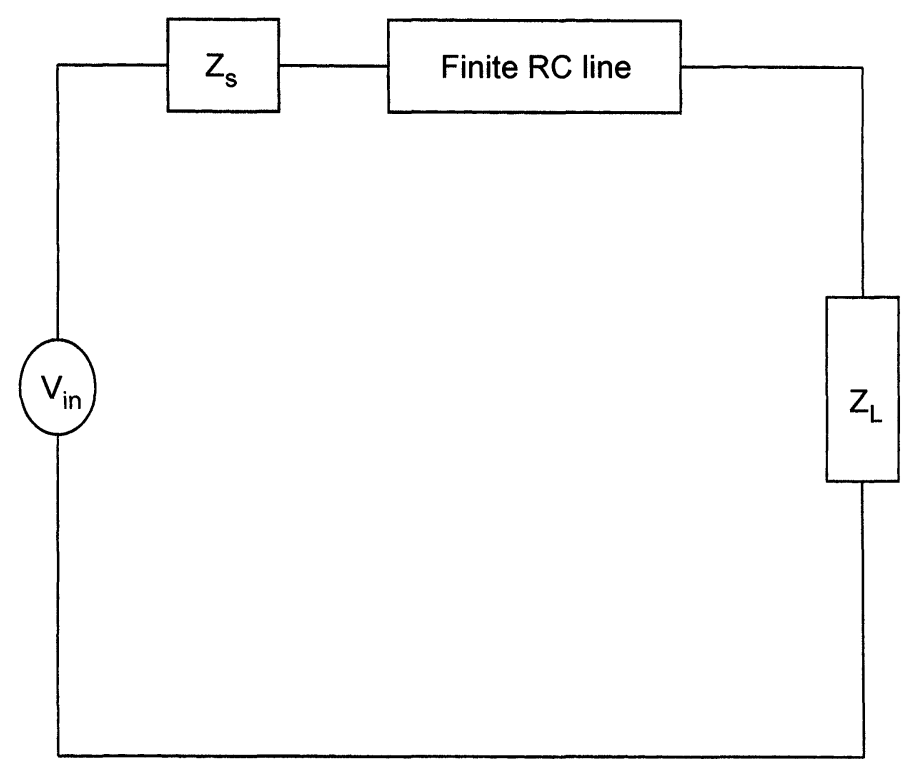

FIGURE 4 The finite RC line. 
where $U_{n}(x, s)$ is the $s$-domain output response at distance $x$ from the source, $V_{n}(x, s)$ is the $s$ domain incident waveform, and $L$ is the length of the finite interconnect. The suffix $n$ indicates that the input is an $n$-th order polynomial of the form $\left(V_{0} / T_{R}^{n}\right) t^{n} u(t)$. The incident waveform is the Laplace transform of the time domain response of a semi-infinite $\mathrm{RC}$ line under $n$-th order polynomial input. This can be obtained using Eqs. (12) and (15) derived in the last sub-section.

The special case of a finite open-ended RC line with ideal source can be easily analyzed. In this case we have, $Z_{s}(s)=0, Z_{l}(s)=\infty$ and so $\Gamma_{l}(s)=1, \Gamma_{s}(s)=-1$.

Therefore from Eq. (16) we have,

$$
\begin{aligned}
U_{n}(x, s)= & V_{n}(x, s) \\
& +\sum_{k=1}^{\infty}\left[(-1)^{k-1} V_{n}(2 k L-x, s)\right. \\
& \left.+(-1)^{k} V_{n}(2 k L+x, s)\right]
\end{aligned}
$$

The corresponding time-domain expression is given by,

$$
\begin{aligned}
u_{n}(x, t)= & v_{n}(x, t) \\
& +\sum_{k=1}^{\infty}\left[(-1)^{k-1} v_{n}(2 k L-x, t)\right. \\
& \left.\quad+(-1)^{k} v_{n}(2 k L+x, t)\right]
\end{aligned}
$$

where $u_{n}(x, t)$ and $v_{n}(x, t)$ are the time-domain finite and semi-infinite RC line responses respectively to $n$-th order input polynomial waveform.

As is evident from Eq. (18), we can directly use the results derived in the last subsection and obtain an expression for the case of finite RC line.

\subsection{Simulation Results}

The equations for the response derived above were simulated in MATLAB. The results obtained have been compared with those obtained through SPICE simulation, in which a uniformly distributed RC model was used for the transmission line. Our results have been found to match with those obtained through SPICE simulation. These results are presented in Figure 5.

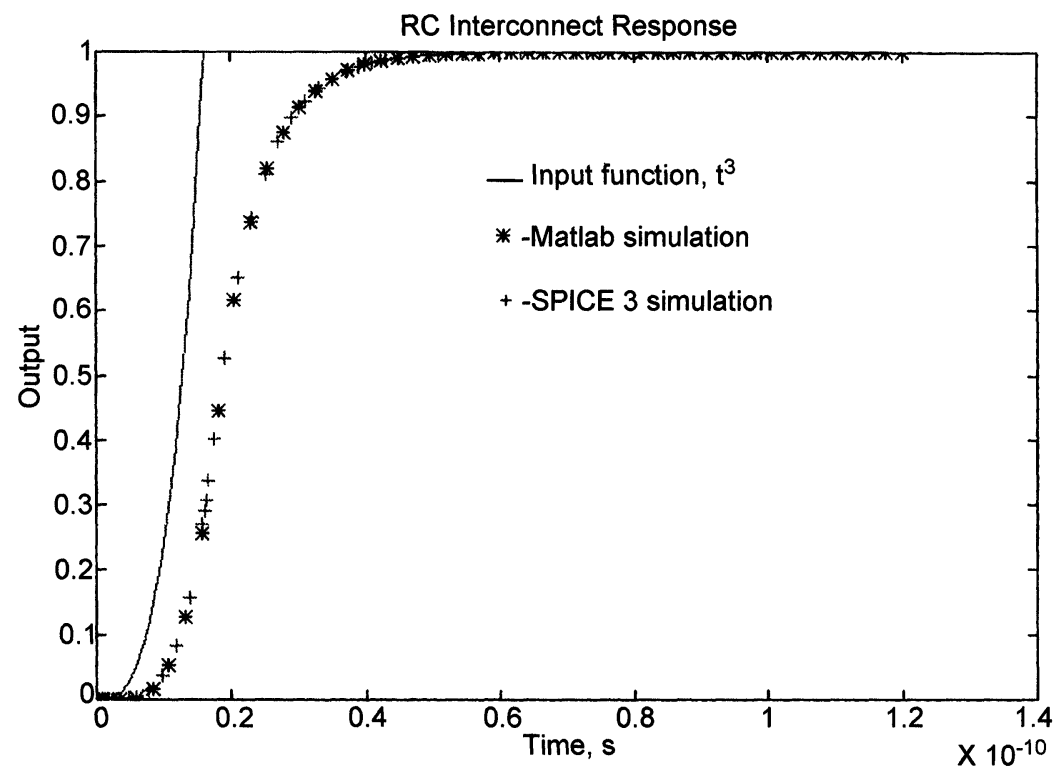

FIGURE 5 Response of RC interconnect to a third order input $\left(t^{3}\right)$. 


\section{CONCLUSIONS}

In this paper we presented analytical expressions for output voltage response of semi-infinite RC lines under polynomial input waveforms. We showed that the output response of a RC interconnect to an $n$-th order polynomial is obtained in terms of the responses to $(n-1)$ st order and $(n-2)$ nd order polynomials. Since the output responses of an RC line for step, ramp and quadratic inputs are easy to derive, these responses can be used to recursively compute the response to an $n$-th order polynomial input. This provides us with a framework to estimate delays of interconnect structures to input waveforms more general than step and ramp. This is important, because in deep sub micron designs input waveshape can play a role in delay estimation.

There is a lot of scope for future work in this area. We need to come up with high-fidelity polynomial approximations to real-life waveforms. This can be done by defining empirical parameters that capture the waveshape of a given waveform faithfully. Then each waveform can be represented by a unique set of these parameters and we can come up with a polynomial fit specific to a waveform by using these parameters. We can feed this polynomial approximation to the $\mathrm{RC}$ interconnect and estimate the output response and delay using the equations derived in the last section. This procedure can be extended to RC trees and we can approximate the waveform at each discontinuity in the tree with polynomial expressions using the parameter set. This leads to the computation of exact output response at all the nodes of a circuit.

\section{Acknowledgments}

The authors would like to thank the reviewers for their valuable suggestions in improving the technical content of this paper. The authors would also like to acknowledge the efforts of Hargovind Singh, T. Preetam Charan Anand and
R. Venkatraman for obtaining the simulation results and in preparing the final manuscripts.

\section{References}

[1] Bakoglu, H. B., "Circuits, Interconnections and Packaging", Addison Wesley, 1990.

[2] Saraswat, K. C. and Mohammadi, F., "Effect of scaling of interconnections on the time delay of VLSI circuits", IEEEJ. Solid-state Circuits, SC-17(2), April, 1982, $275-280$.

[3] Elmore, W. C., "The transient response of damped linear network with particular regard to wideband amplifiers", J. App. Physics, January, 1948, 19, 55-63.

[4] Gupta, R. et al., "The Elmore delay as a bound for RC trees with generalized input signals", ACM/IEEE Design Automation Conference, June, 1995, pp. 364-369.

[5] Kahng, A. B. et al., "Analytical Delay Models for VLSI Interconnects Under Ramp Input", ICCAD, November, 1996, pp. 30-35.

[6] Qian, J. et al., "Modelling the effective capacitance for the RC interconnect of CMOS trees", IEEE Trans. on $C A D$, December, 1994, 13(12), 1526-1535.

[7] Kahng, A. B. and Muddu, S., "Delay analysis of VLSI interconnections using the diffusion equation model", IEEE Design Automation Conference, June, 1994, pp. 563-569.

[8] Kaufman, W. M. and Garrett, S. J., "Tapered distributed filters", IRE Trans. on Circuit Theory, December, 1962, pp. $542-543$.

[9] Kahng, A. B. and Muddu, S., "Analysis of RC interconnects under ramp input", ACM TODAES, April, 1997, 2(2), $168-192$.

[10] Rao, V. B., "Delay analysis of the distributed RC line", ACM/IEEE Design Automation Conference, June, 1995, pp. $370-375$.

[11] Holberg, D. et al., "DC parameterized piecewise function transistor models for bipolar and MOS logic stage delay evaluation", ICCAD, November, 1990, pp. 546-549.

\section{APPENDIX A}

We have defined

$$
I_{0}(x, t)=V_{0} \operatorname{erfc}\left(\frac{x \sqrt{r c}}{\sqrt{4 t}}\right)
$$

It has been shown in [9] that

$$
\begin{array}{r}
I_{1}(x, t)=V_{0}\left[\left(t+\frac{x^{2} r c}{2}\right) \operatorname{erfc}\left(\frac{x \sqrt{r c}}{\sqrt{4 t}}\right)\right. \\
\left.-\frac{x \sqrt{r c}}{\sqrt{\pi}} t^{1 / 2} \mathrm{e}^{-\left(x^{2} r c / 4 t\right)}\right] u(t)
\end{array}
$$


We have,

$$
\begin{aligned}
I_{2}(x, t)= & \int_{0}^{t} I_{1}(x, \tau) d \tau \\
= & V_{0} \int_{0}^{t}\left[\left(\tau+\frac{x^{2} r c}{2}\right) \operatorname{erfc}\left(\frac{x \sqrt{r c}}{\sqrt{4 \tau}}\right)\right. \\
& \left.-\frac{x \sqrt{r c}}{\sqrt{\pi}} \tau^{1 / 2} \mathrm{e}^{-\left(x^{2} r c / 4 \tau\right)}\right] d \tau
\end{aligned}
$$

It is easy to show that

$$
V_{0} \int_{0}^{t} \tau \operatorname{erfc}\left(\frac{x \sqrt{r c}}{\sqrt{4 \tau}}\right) d \tau=t I_{1}(t)-I_{2}(t)
$$

Also, it follows that

$$
V_{0} \frac{x^{2} r c}{2} \int_{0}^{t} \operatorname{erfc}\left(\frac{x \sqrt{r c}}{\sqrt{4 \tau}}\right) d \tau=\frac{x^{2} r c}{2} I_{1}(t)
$$

Let us derive the following integral,

$$
\begin{aligned}
& V_{0} \frac{x \sqrt{r c}}{\sqrt{\pi}} \int_{0}^{t} \tau^{1 / 2} \mathrm{e}^{-\left(x^{2} r c / 4 \tau\right)} d \tau \\
& =-4 \frac{V_{0}}{\sqrt{\pi}} \int_{0}^{(x \sqrt{r c} / \sqrt{4 t})} \tau^{2} \\
& \quad \mathrm{e}^{-((x \sqrt{r c} / \sqrt{4 \tau}))^{2}} d\left(\frac{x \sqrt{r c}}{\sqrt{4 \tau}}\right) \\
& =2\left[t^{2} I_{0}(t)-2 \int_{0}^{t} \tau I_{0}(\tau) d \tau\right] \\
& =2\left[t^{2} I_{0}(t)-2 t I_{1}(t)+2 I_{2}(t)\right]
\end{aligned}
$$

Therefore we have,

$$
\begin{aligned}
& V_{0} \frac{x \sqrt{r c}}{\sqrt{\pi}} \int_{0}^{t} \tau^{1 / 2} \mathrm{e}^{-\left(x^{2} r c / 4 \tau\right)} d \tau \\
& \quad=2\left[t^{2} I_{0}(t)-2 t I_{1}(t)+2 I_{2}(t)\right]
\end{aligned}
$$

Substituting (22), (24) and (25) into Eq. (21) we have,

$$
I_{2}(x, t)=\left(5 / 6 t+\frac{x^{2} r c}{12}\right) I_{1}(x, t)-1 / 3 t^{2} I_{0}(x, t)
$$

So we have proved that

$$
I_{2}(x, t)=\left(k_{1,2} t+k_{2,2}\right) I_{1}(x, t)-k_{3,2} t^{2} I_{0}(x, t)
$$

where $k_{1,2}=5 / 6, k_{2,2}=\left(x^{2} r c / 12\right)$ and $k_{3,2}=1 / 3$

\section{APPENDIX B}

Let us suppose

$$
I_{m}(x, t)=\left(k_{1, m} t+k_{2, m}\right) I_{m-1}(x, t)-k_{3, m} t^{2} I_{m-2}(x, t)
$$

holds for a positive integer $m \geq 2$

Then

$$
\begin{gathered}
I_{m+1}(x, t)=\int_{0}^{t} I_{m}(x, \tau) d \tau \\
=\int_{0}^{t}\left[\left(k_{1, m} \tau+k_{2, m}\right) I_{m-1}(x, \tau)\right. \\
\left.\quad-k_{3, m} \tau^{2} I_{m-2}(x, \tau)\right] d \tau \\
I_{m+1}(x, t)\left(1+k_{1, m}-2 k_{3, m}\right) \\
=\left[\left(k_{1, m}-2 k_{3, m}\right) t+k_{2, m}\right] I_{m}(x, t) \\
-k_{3, m} t^{2} I_{m-1}(x, t)
\end{gathered}
$$

So we have proved that,

$$
\begin{aligned}
I_{m+1}(x, t)= & \left(k_{1, m+1} t+k_{2, m+1}\right) I_{m}(x, t) \\
& -k_{3, m+1} t^{2} I_{m-1}(x, t)
\end{aligned}
$$

where

$$
\begin{aligned}
& k_{1, m+1}=\left(\frac{k_{1, m}+2 k_{3, m}}{1+k_{1, m}+2 k_{3, m}}\right) \\
& k_{2, m+1}=\left(\frac{k_{2, m}}{1+k_{1, m}+2 k_{3, m}}\right) \\
& k_{3, m+1}=\left(\frac{k_{3, m}}{1+k_{1, m}+2 k_{3, m}}\right)
\end{aligned}
$$

\section{Authors' Biographies}

L. M. Patnaik received his Ph.D and D.Sc degrees from the Indian Institute of Science in 1978 and 1989 in the areas of Real-time Systems and 
Computer Architecture respectively. Currently, he is a Professor with the Indian Institute of Science.

His academic interests since 1971 at the IISc have been in the areas of Parallel and Distributed Computing, Mobile Computing, Soft Computing, CAD of VLSI circuits, and Real-time Systems. In these areas, he has published over 330 papers in refereed International Journals and Conference Proceedings. He is Fellow of the IEEE, The Third World Academy of Sciences, and all the four leading Science and Engineering Academies of India. He has been awarded the IEEE Computer Society's 1999 Technical Achievement Award, and more than six prestigeous Indian Research Awards. He serves on the Editorial Boards of almost a dozen International Journals.

Satrajit Gupta is currently a Ph.D student at CMU, he did his M.E. at IISc, Bangalore. His areas of interest are interconnect modelling and analysis for deep submicron VLSI systems. 

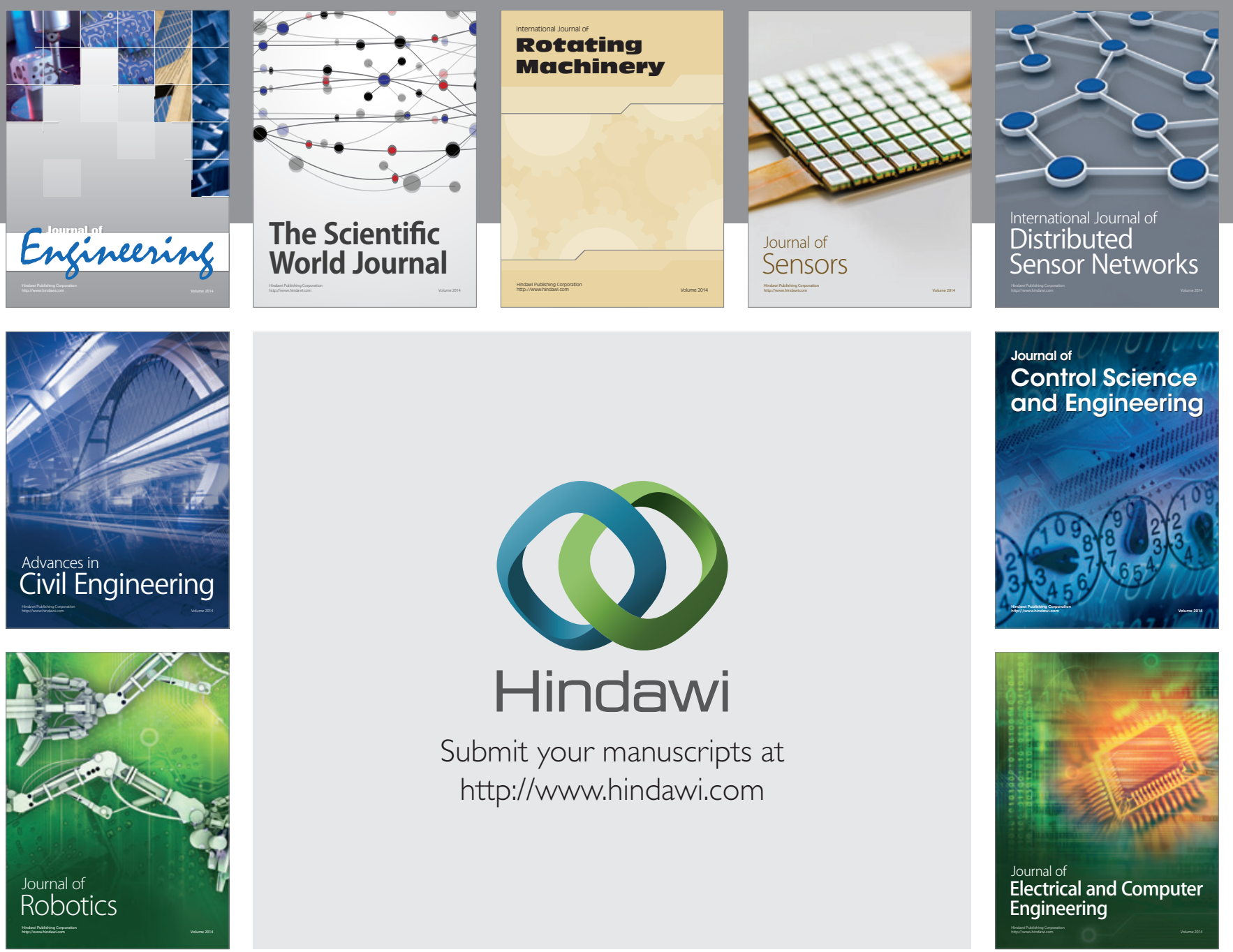

Submit your manuscripts at

http://www.hindawi.com
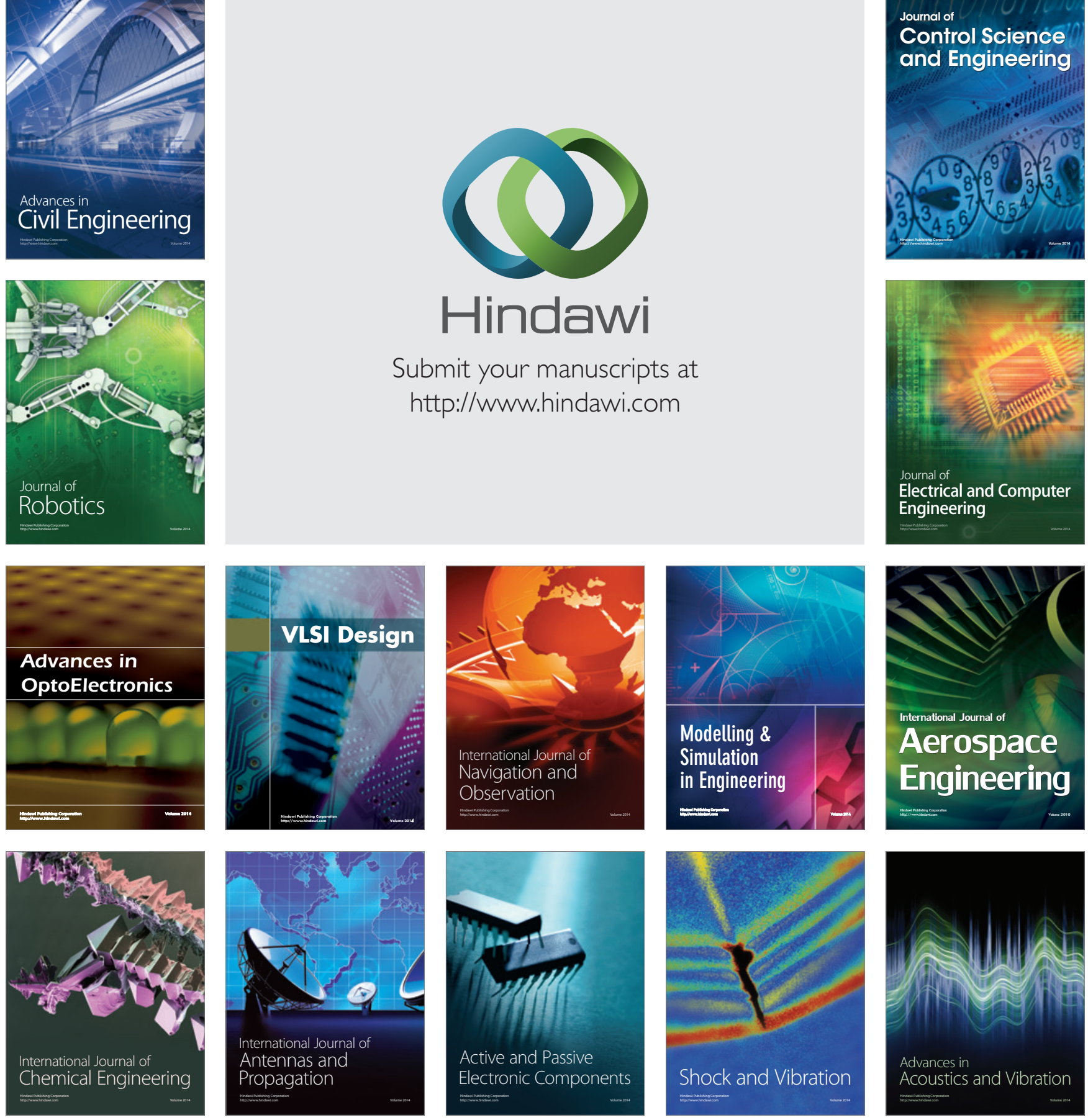